\title{
Endoscopic Surgery of the Spine as a Multi-Disciplinary Subspecialty for Appropriately Trained, and Experienced Providers
}

\author{
Anthony T Yeung ${ }^{1,2^{*}}$, Christopher A Yeung ${ }^{1}$, Nima Salari ${ }^{1}$ and Justin Field ${ }^{1}$ \\ ${ }^{1}$ University of New Mexico School of Medicine, Albuquerque, New Mexico, USA \\ ${ }^{2}$ Desert Institute for Spine Care, Phoenix, Arizona, USA \\ *Corresponding author: Anthony T. Yeung, Desert Institute for Spine Care, Phoenix, Arizona, USA,Tel: +1 602-944-2900; E-mail: ayeung@sciatica.com
}

Rec Date: August 17, 2017; Acc Date: September 08, 2017; Pub Date: September 12, 2017

Copyright: @ 2014 Yeung AT, et al. This is an open-access article distributed under the terms of the Creative Commons Attribution License, which permits unrestricted use, distribution, and reproduction in any medium, provided the original author and source are credited.

\begin{abstract}
Introduction: Endoscopic spine surgery has attracted both surgeons and nonsurgeons in increasing numbers as endoscopic spine systems, a variety of spine endoscopes, and new and evolving surgical instrumentation are developed. The procedure, using fluoroscopically guided percutaneous techniques, are getting more standard, easier, safer, readily reproducible, and more cost effective. It has also been an avenue for surgeons and a few appropriately trained and certified non-surgeons to participate in a minimally invasive, procedure oriented health care delivery platform that provides cost effective results after failure of nonsurgical methods. Such a multidisciplinary team has been established at the University of New Mexico through a donation to the University by the first author.
\end{abstract}

Discussion: Asia, especially China and Korea, has seen adoption of endoscopic spine surgery grow exponentially in the past few years, recognizing that endoscopic spine surgery may be the answer to delivering cost effective spine care to their working and aging population. Two basic methods are the mainstay of current endoscopic techniques. The least invasive techniques in the lumbar spine are transforaminal, but translaminar endoscopic approaches are better accepted and easier for endoscopic surgeons to grasp.

Conclusion: Endoscopic spine surgery has great promise in countries with looking for cost effective delivery of health care to its population. Endoscopic surgery is the least minimally invasive surgical platform that will facilitate a move away from fusion as a first line of surgical treatment, delaying or eliminating fusion for patients who may have indications for decompression and fusion, but do well with an earlier and staged procedure that will mitigate the need for open decompression and fusion by $75 \%$, derived by large individual and group databases known to this author.

Keywords: Endoscopic; Percutaneous; Translaminar; Transforaminal; Decompression; Ablation; Endoscopic Pathoanatomy; Patient indication stratification

\section{Introduction}

Endoscopic spine surgery attracts both surgeons and non-surgeons in increasing numbers as endoscopic spine systems, a variety of spine endoscopes, and new and evolving surgical instrumentation develop. The procedure, using fluoroscopically guided percutaneous techniques, is getting more standard, easier, safer, readily reproducible, and more cost effective. It is an avenue for surgeons and a few appropriately trained and certified non-surgeons to participate in a minimally invasive, procedure oriented health care delivery platform that provides cost effective results after failure of nonsurgical methods. Such a multi-disciplinary team is established at the University of New Mexico through a donation to the university by the first author. The non-surgeons will work as a multidisciplinary team that will share responsibilities according to their certification and training. Endoscopic techniques create less surgical morbidity than traditional open surgical methods. Adequate training, however, is lacking for both specialty providers, but especially for non-surgeons. A focus on Robotics specifically for endoscopic spine surgery, currently under development, may provide the surgical support and impetus to bring this technique more rapidly to mainstream surgery.

Patients educate themselves by researching treatment options for their ailments on the Internet, but it is difficult to find truly competent and experienced providers. Many advertise minimally invasive surgery to attract patients, but only a few have extensive experience or true expertise. Both good surgeons and non-surgeons unfamiliar with the new techniques are unable to guide their patients because of lack of actual experience, but only exposure to the online and published benefits of endoscopic spinal surgery. Non-surgeons are especially vulnerable to lack of adequate training and may create greater surgical risk to their patients.

Experienced endoscopic spine surgeons with surgical expertise are driving patients to endoscopic spine surgery by their results. These surgeons have become greatly sought after by patients and referring physicians who have experienced the benefits of endoscopic spine surgery. Patients seek them out. The demand currently exceeds the capacity of a limited number of highly qualified and successful endoscopic surgeons who have not just achieved proficiency, but have truly embraced this minimally invasive surgical platform as their surgical focus. While many of these surgeon's tout level $\mathrm{V}$ expert opinion of "evidence" based medicine, the " $E$ " in "evidenced based" should also be backed by "experience based medicine", with experience 
and proficiency being the most important surgeon factor for the patient to consider. The "E" may not only stand for endoscopic, evidence, and experience based, but "economically" based. Unfortunately, many payers are not familiar with this platform, and do not provide appropriate payment insurance guidelines for this effective surgical procedure and thus their reimbursement, if at all, may not be sufficient to allow endoscopic surgery to flourish. The costs of specialized equipment such as lasers, disposables, and other specialized instruments that facilitate endoscopic surgery becomes unsustainable without adequate reimbursement, thus further restricting the adoption of endoscopic spine surgery as a cost effective beneficial manner of delivering health care when only motivated cash pay patients are available.

By focusing on, and identifying the patho-anatomy of the pain generator as opposed to only using imaging to identify abnormalities on X-ray, CT scan, or MRI, a surgeon who is able to identify the pathoanatomy of pain and correlate these findings with the pathophysiology of pain will provide cost effective spine care. By stratifying patient selection and by performing their own diagnostic and therapeutic injections, surgeons can predict the results of endoscopic decompression, ablation, and irrigation. The evidence based research demonstrates patients attain successful results with a good to excellent rating over two to ten years. This evidence-based information comes from large group and individual databases like that at Desert Institute for Spine Care in Phoenix, Arizona. This form of big data has opened the door to new endoscopically based level $\mathrm{V}$ evidence touted by spine societies, through documentation of patho-anatomy when treating the pain generator for this sought-after subspecialty.

Two basic methods are the mainstay of current endoscopic techniques. The least invasive techniques in the lumbar spine are transforaminal, but translaminar endoscopic approaches are better accepted and easier for endoscopic surgeons to grasp. Anthony Yeung pioneered the "inside out" Yeung Endoscopic Spine System (YESSTM), a trademarked transforaminal technique, while Thomas Hoogland, (now deceased) pioneered the "outside-in" Thessys system by Joimax. Hoogland later modified his system to a system known as the Maxmore system and technique to trephining and reaming the inside the walls of the access cannulas favored by Yeung. Further modifications also create a "targeted" hybrid evolved by Yeung and adopted by others in Korea and China as key opinion leaders supporting competing endoscopic companies. Hoogland and other prominent endoscopic surgeons still performed the majority of his early patients with the translaminar approach under general anesthesia, even at L5-S1, while Yeung has special instruments and foraminoplasty techniques to reach the epidural space with the transforaminal approach, including L5-S1. The endoscopic surgical technique has evolved and expanded to the cervical and thoracic spine and for treatment of conditions previously used only in open surgical techniques with more surgically invasive and surgically morbid procedures. The endoscopic techniques also include "full" endoscopic techniques favored by Ruetten, but with emphasis on the translaminar endoscopic approach under general anesthesia.

Multiple publications in peer reviewed subscription and open access journals are increasing exponentially, and validating this minimally invasive endoscopic procedure through publications in recent years.

Asia, especially China and Korea, has seen adoption of endoscopic spine surgery grow exponentially in the past few years, recognizing that endoscopic spine surgery may be the answer to delivering cost effective spine care to their working and aging population. The Chinese
Medical Association recently convened a special meeting attended by surgeons, nonsurgeons, and traditional medicine providers interested in promoting and supporting endoscopic surgery in China to service the needs of its working and aging population. An inaugural meeting was held in Beijing on July 8, 2017 for this purpose. Anthony Yeung was invited to be the keynote speaker.

Endoscopic Spine Surgery has great promise in countries looking for cost effective delivery of health care to its population. Endoscopic surgery is the least minimally invasive surgical platform that will facilitate a move away from fusion as a first line of surgical treatment, delaying or eliminating fusion for patients who may have indications for decompression and fusion, but do well with an earlier and staged procedure that will mitigate the need for open decompression and fusion by $75 \%$, derived by large individual and group databases known to this author.

\section{Literature Review}

\section{Historical review}

Over the years, traditional laminectomy and discectomy has undergone modification, first through smaller openings-in the form of a laminotomy/laminectomy and discectomy. This process was made minimally invasive by micro discectomy utilizing magnification and smaller incisions [1-5].

Arthroscopic micro discectomy, a term coined by Parviz Kambin, aided by expanded use of spinal endoscopy now give spine surgeons the ability to visualize spinal structures and the patho-anatomy of pain generators not previously possible with open surgery. In addition, the use of local anesthesia helps the endoscopic surgeon correlate pain generation and pain stimulation as well as pain relief reported by the patient during the surgical procedure.

\section{The birth of modern transforaminal endoscopic decompression}

The use of a multichannel flow integrated spine scope originally developed by A Yeung as the Yeung Endoscopic Spine system, $\left(\right.$ YESS $\left.^{\mathrm{TM}}\right)$ and technique made visualization inside the disc an integral part of visualized intradiscal therapy [6,7], but also promoted visualization of the foramen and epidural space.

Other endoscopes and competing pioneering surgeons with their own techniques have also evolved endoscopic spine surgery, concomitant with different techniques by different key opinion leaders, aided by evolving instrumentation [8-10]. This has allowed the surgeon to address the full spectrum of painful degenerative and traumatic conditions on the spine, including spine tumors.

Advanced endoscopic surgeons can now remove not just herniated disc material, but clearly visualize, probe, document and correlate other painful spinal conditions with its response to various stimulations. The surgeon can treat painful conditions further by utilizing other forms of intradiscal therapy available, such as laser, radio frequency, nucleus augmentation and biologics [11,12]. Not all techniques and equipment are equivalent, therefore all endoscopic surgeons and non-surgeons are in encouraged to learn from the different "Masters".

The ability to perform these visualized minimally invasive procedures not only gives surgeons a method to evaluate and treat a 
variety of painful conditions in patients not responding to more traditional conservative, noninvasive techniques, but it augments already established open, validated methods. This includes selected cases of degenerative and isthmic spondylolisthesis, considered most appropriate for fusion, but fusion can be either delayed or altogether eliminated by slowing the pain from the degenerative process in carefully selected, stratified patients [13]. Endoscopic techniques can vary and will likely depend on the "surgeon factor" in obtaining favorable results. The ability to evaluate the area in and around the foramen and nerves opens a whole new dimension for the evaluation and treatment of common and complex back pain. Access to spinal pathology may also vary in individual patients, and special techniques may need to be utilized to gain access to the pathologic lesion and to avoid injury to spinal structures. Radiologic considerations in each patient and anatomic variations will determine whether the disc space and/or spinal canal are accessible. Surgical risks remain, and the patient must accept these risks.

\section{Discussion}

At a meeting of the Asian Minimally Invasive Spine Society in Bangkok, July 2017, Dr. Bin Zhu from Peking University's Third Hospital (PUTH) the largest spine hospital in China, who has performed $>500$ spine cases/year, reported on complications from PELD discussion groups in China. These complications were not reported publicly or in the literature [14]. China has traditional medicine and non-surgeons who often take up new techniques independently without formal training. Surgeons are employed by public institutions and do not compete with one another as may be the case in the West. One death was reported due to lack of training and an inadvertent placement of a surgical cannula and instruments to the abdomen, causing a death by inadvertent injury to abdominal vessels. Other complications, reported and unreported have occurred from visceral injuries, infection, nerve injuries, and failure to adequately remove the patho-anatomy $[15,16]$. While complications can occur with every surgical procedure, it is imperative that these actual and potential complications that can happen with surgical procedures are recognized by the operating surgeon. Adequate training is imperative to not allow the technique to fall into disrepute. These adverse events emphasize proper training for all providers, surgeon and non-surgeon alike who will embrace the responsibility to make this evolving minimally invasive procedure safe, cost effective, and beneficial to patients of all countries [17]. I am aware of other risks when I am made aware of complications in medical legal matters. Non-surgeons who get sued for complications are more vulnerable if the patient is not informed that their surgeon is not a board-certified surgeon by training.

The learning curve is both steep and shallow, often requiring 5 years to become proficient and exceptional. A fellowship or preceptorship with experienced endoscopic spine surgeons is recommended for surgeons who want to excel and take advantage of all the capabilities of spinal endoscopy $[18,19]$. Non-surgeons wanting to incorporate advancements in endoscopic spine surgery in their field of specialization must also have adequate training and background experience. This is best practiced in a multi-disciplinary environment since surgical and nonsurgical training is different, medical and surgical background is different, concepts are different, and training is different. Technical abilities are also different without formal training. Because of this, the surgeon should take the lead in the ideal multidisciplinary environment, and each stakeholder will naturally evolve by working together rather than be concerned about "protecting their turf" when working together and using all the individual skills for treating their mutual patients.

Painful degenerative conditions of the lumbar spine as described by Pfirmann are especially valuable for treatment by endoscopic means (Figure 1).

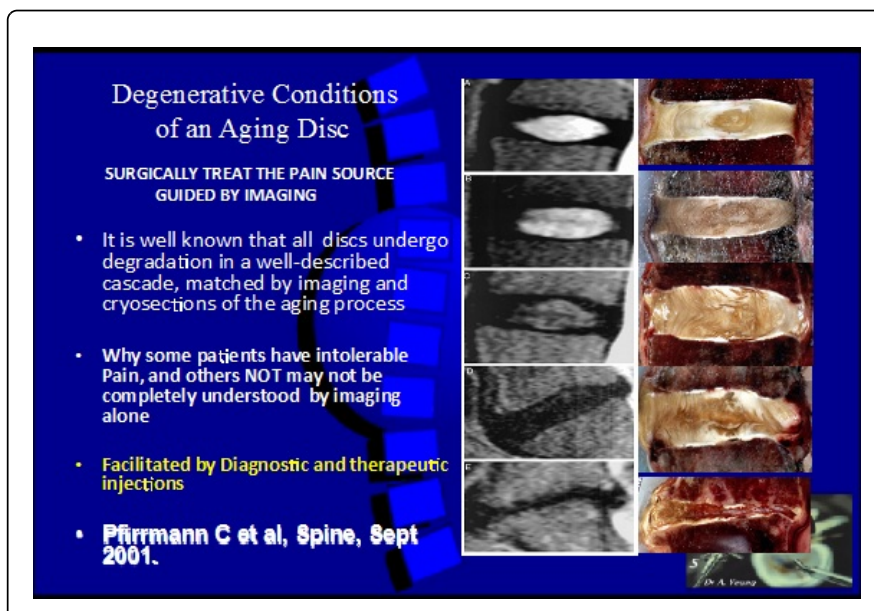

Figure 1: Imaging is only used as a guide. Other means of identifying the pain generator incorporate diagnostic and therapeutic injections augmented by visualization of painful pathoanatomy will override imaging available.

Endoscopic spine surgery will provide the least minimally invasive surgical solution for our patients' spinal pain. The future of endoscopic surgery embraces. "Surgery" not just for a neurologic deficit, an "abnormal" imaging study, or "last resort" for surgical intervention, but targets the patho-anatomy and patho-physiology of pain. Transforaminal endoscopic decompression will therefore be for "surgical pain management".

\section{Transforaminal endoscopic minimally invasive surgery vs. pain management}

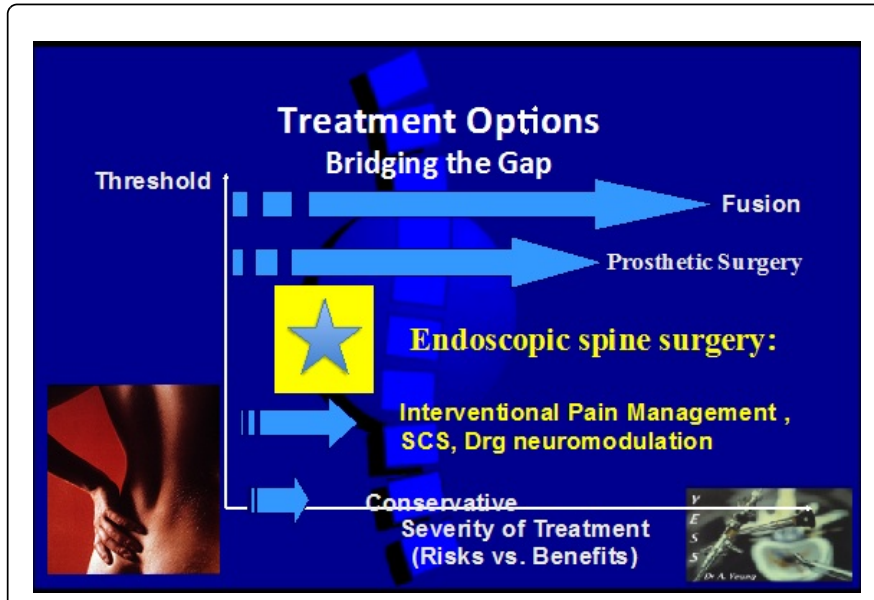

Figure 2: Treatment options in the spectrum of spine care from non-surgical to end result fusion. 
Citation: Yeung AT, Yeung CA, Salari N, Field J (2017) Endoscopic Surgery of the Spine as a Multi-Disciplinary Subspecialty for Appropriately Trained, and Experienced Providers. J Spine 6: 388. doi:10.4172/2165-7939.1000388

Page 4 of 4

Endoscopic spine surgery is not taught in traditional spine surgery or pain management programs. A cooperative program in academic centers may be required to "bridge the gap" between the different stakeholders (Figure 2).

\section{Conclusion}

Endoscopic spine surgery has great promise in countries looking for cost effective delivery of health care to its population. Endoscopic surgery is the least minimally invasive surgical platform that will facilitate a move away from fusion as a first line of surgical treatment, delaying or eliminating fusion for patients who may have indications for decompression and fusion, but do well with an earlier and staged procedure that will mitigate the need for open decompression and fusion by $75 \%$, derived by large individual and group databases known to this author.

The future in spine care may well need to be a joint effort for all specialties and stake holders to cooperate and to safely bring this promising treatment platform for the working and aging populations of the world. Endoscopic spine surgery may be one answer to lowering overall health care costs by eliminating duplication and "unnecessary treatment" for the sake of patient safety and cost effectiveness.

\section{References}

1. Yeung AT (2017) Delivery of spine care under health care reform in the United States. J Spine 6: 372 .

2. Zhang X, Du J, Yeung AT (2017) Development of Percutaneous Endoscopic Lumbar Discectomy (PELD) Technology in China. J Spine 6: 374.

3. Yeung A, Yeung CA (2017) Endoscopic identification and treating the pain generators in the lumbar spine that escape detection by traditional imaging studies. J Spine 6: 369.

4. Yeung AT (2017) A short note on minimally invasive lumbar spine surgery. J Spine 6: e127.

5. Yeung AT (2017) Transforaminal endoscopic decompression for painful degenerative conditions of the lumbar spine: A review of one surgeon's experience with over 10,000 cases since 1991. J Spine Neurosurg 6: 2.

6. Yeung AT, Gore SR (2001) Evolving methodology in treating discogenic back pain by Selective Endoscopic Discectomy (SED) and thermal annuloplasty. J Minim Invasive Spine Surg Tech 1: 8-16.
7. Gore SR, Yeung AT (2003) Identifying sources of discogenic pain. J Minim Invasive Spine Surg Tech 3: 21-24.

8. Yeung AT, Tsou PM (2002) Posterolateral endoscopic excision for lumbar disc herniation: surgical technique, outcome, and complications in 307 consecutive cases. Spine (Phila Pa 1976) 27: 722-731.

9. Yeung AT (2000) Minimally invasive surgery with the Yeung endoscopic spine system (YESS). Surg Tech Int 8: 267-277.

10. Tsou PM, Yeung AT (2002) Transforaminal endoscopic decompression for radiculopathy secondary to intra-canal non-contained lumbar disc herniations: outcome and technique. Spine Journal 2: 41-48.

11. Yeung AT, Yeung CA (2003) Advances in endoscopic disc and spine surgery: The Foraminal Approach. Surgical Tech Int 11: 253-261.

12. Yeung AT, Yeung CA (2006) In-vivo endoscopic visualization of pathoanatomy in painful degenerative conditions of the lumbar spine. Surg Technol Int 15: 243-256.

13. Yeung AT (2007) The evolution and advancement of endoscopic foraminal surgery: One surgeon's experience incorporating adjunctive technologies. SAS J 1: 108-117.

14. Yeung AT, Gore SR (2011) In-vivo endoscopic visualization of pathoanatomy in symptomatic degenerative conditions of the lumbar spine II: Intradiscal, foraminal, and central canal decompression. Surg Technol Int XXI: 299-319.

15. Hermantin FU, Peters T, Quartararo L, Kambin P (1999) A prospective randomized study comparing the results of open discectomy with those of video-assisted arthroscopic microdiscectomy. J Bone Joint Surg 81: 958-965.

16. Ruetten S, Komp M, Merk H, Godolias G (2008) Full-endoscopic interlaminar and transforaminal lumbar discectomy versus conventional microsurgical technique: A prospective, randomized, controlled study. Spine (Phila Pa 1976) 33: 931-939.

17. Yeung AT, Yeung CA, Salari N, Field J, Navratil J, et al. (2017) Lessons learned using local anesthesia for minimally invasive endoscopic spine surgery. J Spine 6: 377.

18. Yeung AT (2017) Robotics in the MIS spine surgery arena: A new role to advance the adoption of endoscopic surgery as the least invasive spine surgery procedure. J Spine 6: 374.

19. Anthony TY, Christopher AY (2017) Selective Endoscopic Lumbar Discectomy $\left(\mathrm{SED}^{\mathrm{sin}}\right)$ and thermal annuloplasty for discogenic back pain, disc herniations and sciatica in high performance athletes and physically active patients. Sports Injr Med 2017: 110. 